\title{
Le Nouveau Partenariat Pour Le Développement De l'Afrique (Nepad) : Maternité Difficile Et Echec Processuel
}

\author{
Kayibu Becker Igor, Kitwa Kalenga Matthieu, Bwanga Anembali Blaise. \\ Assistants à la Faculté de Droit de l'Université de Likasi, République Démocratique du Congo.
}

\begin{abstract}
At its creation on May 24, 1963, the Organization of African Unity (OAU) had been assigned two main objectives: the development and the total decolonization of Africa. However, in order to achieve development, the OAU will put in place a development strategy: the Lagos Plan of Action (PAL) with the aim of making Africa accessible to development through the establishment of an African Economic Community. But several years after its creation, the OAU, on its two main objectives; only decolonization had succeeded but not development. Hence, it will appeal to African leaders to replace the OAU with the African Union in 2001. Faced with the failure of the LAP, a development strategy will be designed in the context of the African Union: NEPAD.Unfortunately, NEPAD did not achieve its development objective within the deadline set for two main reasons: the lack of communication of its real scope to the main beneficiaries (African actors) and the lack of its own financial resources.
\end{abstract}

\section{RESUME}

A sa création le 24 Mai 1963, l'Organisation de l'Unité Africaine (OUA) s'était vu assigner deux objectifs principaux à savoir : le développement et la décolonisation totale de l'Afrique. Cependant pour aboutir au développement l'OUA mettra en place une stratégie de développement : le Plan d'Action de Lagos (PAL) avec pour but de faire accéder l'Afrique au développement par la réalisation d'une Communauté Economique Africaine. Mais plusieurs années après sa création, l'OUA, sur ses deux objectifs principaux; seule la décolonisation avait réussi mais pas le développement. D'où, il plaira aux dirigeants africains de remplacer l'O.U.A par l'Union Africaine en 2001. Face à l'échec du PAL, il sera ainsi conçu une stratégie de développement dans le contexte de l'Union Africaine : Le NEPAD. Malheureusement le NEPAD, n'a pas réalisé son objectif de développement dans l'échéance lui imparti pour deux raisons majeures : le déficit de la communication de sa portée réelle aux principaux bénéficiaires (acteurs africains) et la carence des ressources financières propres.

\section{INTRODUCTION}

La réflexion sur la politique économique africaine n'a jamais mobilisé autant d'énergies comme ces dernières années dans le seul souci de démontrer et d'expliquer les contre-performances alarmantes de l'Afrique. C'est «la région du monde, où presque la moitié de la population vit dans une pauvreté absolue, accentuée par la famine et la malnutrition, alors que ses ressources naturelles réjouissent les autres calmement. C'est la région où environ $70 \%$ de la population vit dans le noir ou s'éclaire en recourant au feu du bois ou à la lampe à pétrole ». ${ }^{1}$

C'était ce portrait flou de l'Afrique que le NEPAD s'entendait effacer. Dès lors que des projets antérieurs avaient lamentablement échoués entre autres : le plan d'action de Lagos (PAL) de 1980, le consensus de Washington ou les plans d'ajustement structurels, les cadres d'études des perspectives à long terme etc. Tous ces projets n'ont pas réussi à stopper la dégradation économique des années 1980 et qui s'est amplifié vers les années 1990.Cette pléthore d'initiatives va ainsi ouvrir la voie à la Nouvelle Initiative Africaine (N.I.A) qui s'est muée en Juillet 2001 en Nouveau Partenariat pour le Développement de l'Afrique (NEPAD).

\section{LE NEPAD : SENS ET OBJECTIFS}

Le NEPAD est né de la fusion du Millenium African Plan (MAP) proposé par OLUSEGUN OBASANJO et ABDELAZIZ BOUTEFLIKA et du Plan OMEGA D'ABDOULAYE WADE, dénommé d'abord « Nouvelle Initiative Africaine » (N.I.A.) pour plus tard être baptisé NEPAD. Le NEPAD tel qu'il se présente n'est rien d'autre que « une promesse faite par des dirigeants Africains fondée sur une vision commune. Ainsi qu'une conviction ferme et partagée qui leur

${ }^{1}$ www.nouvellesdunepad.com, consulté le 28 septembre 2011 
incombe d'urgence d'éradiquer la pauvreté, de placer leurs états individuellement et collectivement, sur la voie d'une croissance et d'un développement durable, tout en participant activement à l'économie et à la vie politique $»{ }^{2}$

En définitive « le NEPAD est compris dans le contexte de l'Union Africaine. Il est donc l'interprétation et la programmation claire du développement et de l'unité de l'Afrique ${ }^{3}$

\section{Les objectifs du NEPAD ${ }^{4}$ sont:}

- Assurer une croissance annuelle du PIB de plus de 7\% et s'y maintenir,

- Faire en sorte que le continent réalise les objectifs convenus en matière de développement international.

- Réduire de moitié de 1990 à 2015 le pourcentage d'extrême pauvreté

- Assurer la scolarisation de tous les enfants en âge de fréquenter les écoles primaires d'ici 2015.

- Scolariser un grand nombre des femmes et filles d'ici 2005.

- Réduire le taux de mortalité infantile et post-infantile de 2/3 de 1990 à 2015.

- Réduire le taux de maternité de 3/4 de 1990 à 2015.

- L'accès intégral à des services de santé génétique d'ici 2015.

- Mettre en œuvre dès 2005 des stratégies régionales de développement durable pour que les pertes des ressources écologiques soient compensées.

Tous ces objectifs ont été élaborés en vue « d'aboutir à un projet commun qui permettra à l'Afrique de récupérer son retard de développement par rapport aux autres continents de la planète. Surtout en ce qui concerne la croissance économique, le développement des emplois, la diversification des activités de production, l'amélioration de la compétitivité sur le plan international, l'augmentation des exportations, la réduction de la pauvreté et des inégalités ${ }^{5}$

\section{LE NEPAD FACE AUX ORGANISATIONS REGIONALES AFRICAINES}

L'union Africaine est le résultat de la quête pour l'unité et le développement de l'Afrique. «Sous l'égide de l'OUA, les Pays africains ont pris un certain nombre d'initiatives dans des nombreux domaines ce qui a ouvert la voie à la création de l'Union Africaine $»{ }^{6}$

Le NEPAD s'est inspiré de la subdivision géographique du Plan d'Action de Lagos dont : l'Afrique du Nord avec l'Union du Maghreb Arabe (UMA), l'Afrique de l'Ouest avec la Communauté Economique des Etats de l'Afrique de l'Ouest (CEDEAO), l'Afrique de l'Est avec la Communauté du Marché Commun de l'Afrique Centrale et de l'Est (COMESA), l'Afrique Centrale avec la Communauté Economique des Etats de l'Afrique Centrale (CEEAC) et l'Afrique Australe avec la Communauté pour le Développement Economique de l'Afrique Australe (SADC).

Mais en réalité les processus d'intégration au demeurant très inégaux ne sont pas effectués selon ce découpage géographique.

\section{LE NEPAD FACE AUX PAYS INDUSTRIALISES, LES INSTITUTIONS DE} BRETTON WOODS ET L'ONU

De la colonisation à ce jour, il s'avère que l'occident n'a jamais œuvré pour le développement de l'Afrique. Bien qu'il ait existé « une brève période agrémentée d'heureux rapports économiques entre l'Europe et l'Afrique entre 1975 et 1994, qui s'inscrivaient dans le cadre d'accords quinquennaux de coopération pour le développement connus sous le nom de la convention de Lomé $\gg^{7}$ Il est certes, vrai que cela n'avait pas plu aux Etats Unis suite à sa faiblesse politique de l'époque, l'Europe également s'était rebiffée en 1994 à l'issue des négociations de l'Uruguay Rounds.

\footnotetext{
${ }^{2}$ Nouveau partenariat pour le développement de l'Afrique, Octobre 2001, P.1

${ }^{3}$ ESAMBU MATENDA A.B., "Le nouveau partenariat pour le développement de l'Afrique (NEPAD) : un mythe ou une réalité développementalisant dans le contexte de l'Union Africaine " in CAHIER CONGOLAIS D'ETUDES POLITIQUES ET SOCIALES, n 25, Juin 2005, PUL, P. 155

${ }^{4}$ Nouveau Partenariat pour le Développement de l'Afrique, Octobre 2001, P.2.

${ }^{5}$ WENU BECKER, Les organisations internationales africaines et la problématique de la sécurité en Afrique. Praxis de la modélisation d'une approche sécuritaire africaine, thèse de doctorat en R.I., UNILU, 2003-2004, P. 204

${ }^{6}$ DIOUF A.M, La quête de l'Union et le développement Africain, Paris, L'harmattan, 2003, P. 126.

${ }^{7}$ HUGON PHILIPPE, L'économie de l'Afrique, Paris, Ed. La découverte, 2000, P. 6

DOI: 10.9790/0837-2203070509 www.iosrjournals.org 6 Page
}


Il serait irréaliste d'envisager actuellement le développement de l'Afrique par l'apport d'un pays étranger parce que dans les relations économiques internationales le mercantilisme passe pour la morale. Surtout que l'occident et l'orient s'active à créer un réseau d'Etats Africains à leur solde dans le seul but de fragiliser les relations commerciales intra-africaines en lieu et place de l'encourager et de renforcer sa dynamique vers l'intégration régionale. Pour preuve le NEPAD sollicite les subventions agricoles de l'union Européenne dans l'intention de voir cette dernière influencer à son tour positivement les signataires de l'Accord Afrique-Caraïbes-Pacifique (ACP) en vue de lui ouvrir leurs marchés.

La véritable image de l'organisation mondiale du commerce aujourd'hui est celle d'une institution constituée au seul profit de l'Union Européenne, des Etats Unis, du FMI et de la Banque Mondiale. Même lors des assises de l'Organisation Mondiale du Commerce tenus respectivement à Seattle en 1999 et à Doha en 2001, les Etats Unis et l'Union Européenne vu que les prévisions de l'OMC et des Etats de l'ACP contrastaient avec leurs attentes, ils boycotta la rencontre prochaine en s'appuyant sur les accords de libre échange datant de 1998.

Parce que par la suite, l'Union Européenne et les Etats Unis s'étaient réjouis de voir l'OMC maintenir seules les subventions agricoles à leur bénéfice. Pourtant, les pays en voie de développement ont aussi droit aux marchés Européens conformément aux normes de l'OMC.

C'est curieusement auprès de cette organisation que le NEPAD va solliciter de l'aide quoi de plus normal pour tout analyste averti de n'offrir aucune chance à pareille démarche partant des raisons évoquées ci-haut. Les multiples déclarations de l'Ex-Président Français Nicolas Sarkozy aux différentes assises de l'ONU, valaient tout leur sens. Par ce canal il invitait la communauté Internationale à éviter les erreurs du passé envers l'Afrique au risque de ne pas assister à une autre crise financière internationale d'ici 2030.

Le NEPAD aujourd'hui affiche l'image d'une stratégie inféodée à l'occident du fait que les Chefs d'Etats et des Gouvernements africains se confirment de plus en plus comme des pions et vassaux de grandes puissances, des multinationales et des institutions financières internationales.

Lorsqu'ont parcourt « l'acte constitutif du NEPAD, il est prévu d'attendre un financement des institutions de Bretton Woods $»^{8}$. Bien que ces dernières aient toujours nourrit une hostilité face à la gestion souveraine des institutions politiques africaines.

\section{LE NEPAD FACE A LA DEMOCRATIE, LA BONNE GOUVERNANCE ET L'ATTITUDE DES DIRIGEANTS AFRICAINS}

Sous l'influence du vent de la démocratisation ayant soufflé au début des années 1990 sur l'Afrique « tous les partenaires étatiques et non étatiques extérieurs, avant d'accorder de l'aide exigent un certain nombre des conditions dont la démocratie, la bonne gouvernance, la privatisation des entreprises publiques etc.» ${ }^{9}$

D'où la présence des certains instruments juridiques comme la charte africaine des droits de l'homme et des peuples reprenant certaines de ces conditions.

Cependant les chances de tous ces textes parfois élogieux ont souvent été amoindris par la réalité politique africaine, « le calcul est simple, sur 100\% des dirigeants africains qui se précipitent soit pour élaborer, soit pour ratifier certains instruments juridiques prônant la démocratie et la bonne gouvernance, 65\% sont des dictateurs, $20 \%$ des dirigeants des démocraties des façades et $15 \%$ des démocrates $\gg{ }^{10}$.

Curieusement tous ces dirigeants prétendent prêter main forte au NEPAD tout en ignorant qu'ils ne sont pas des modèles en matière de gouvernance. La société mondialisée aujourd'hui est caractérisée par l'influence de la montée en puissance des multinationales. Actuellement les plus puissantes sociétés multinationales contrôlent $25 \%$ du commerce Mondial. Pendant que les dirigeants africains eux ont opté pour l'achat des consciences et le clientélisme politique. Le souci pour l'Afrique, c'est d'avoir des dirigeants ayant la maitrise des enjeux internationaux de l'heure. Ainsi sa maternité difficile surgit par le fait que cet état des choses s'apparente à une malformation congénitale ; en effet le projet du NEPAD est naît de l'échec du Plan d'Action de Lagos qui a souffert des mêmes tumultes, qui lui sagement couvées et entretenues par des dirigeants dictateurs et despotes. Or le développement d'un continent est d'abord une question nationale. Au cas contraire, ce projet serait nait de l'assemblage des différentes misères africaines. A notre avis il serait réfléchi de remodeler les stratégies du Plan d'Action de Lagos après son échec cuisant que de le subroger par le NEPAD mal né. Quant à son échec processuel il se justifie par le fait que les concepteurs du NEPAD, l'ont conçu à la seule fin de mendier. C'est ainsi qu'ils ont réussi à le faire dédouaner auprès des certaines institutions comme le G8 et le FMI.

\footnotetext{
${ }^{8}$ KOFFI ANNAN, Rapport sur les causes des conflits et la promotion d'une paix et d'un développement durable en Afrique, 2003, P.9

${ }^{9}$ HARSH E., " Le nouveau partenariat de l'Afrique " in Afrique Relance, $\mathrm{n}^{\circ}$ 16, Février 2003, P.7

${ }^{10}$ PLATTARD Y., "L'Afrique trente ans après " in $\underline{\text { Revue de deux mondes, }} \mathrm{n}^{\circ}$ 59, Septembre 1991, P.83
} 


\section{CRITIQUES ET PERSPECTIVES}

\section{a. Critiques}

Le NEPAD est effectivement né des cendres d'une kyrielle d'initiatives ayant fait piètre figure. A la seule différence que le NEPAD relève plus d'une vision politique que d'une vision de développement, et la question de sa traduction en termes opérationnels se pose. Dans l'état actuel, les modalités pratiques restent imprécises et ne permettent pas de discerner les priorités spécifiques dans les investissements à réaliser. Le programme d'action du NEPAD n'est donc pas véritablement une stratégie de développement, mais il s'apparente plutôt à un paquet de vœux pieux et irrationnels.

Tous ces programmes réunissent visiblement les conditions requises pour réaliser un développement durable. Il s'intéresse ensuite à la mobilisation des ressources et à la mise en œuvre du nouveau partenariat. Seulement que ce projet n'avait pas été diffusé jusqu'à la base. Aussi les dispositions du NEPAD ne prévoient aucune stratégie de communication à l'endroit de ses vrais destinataires (africains).

Cette situation est de nature à affaiblir le processus d'appropriation du profit à l'échelle du continent. Le NEPAD a connu une entorse un jour après sa création ; «les pionniers de ladite initiative se sont précipitées pour aller en tenir explication aux occidentaux et à l'Union Européenne... pendant que les Africains eux-mêmes jusqu'à lors ne s'étaient pas encore imprégnés de ce que c'est le NEPAD ${ }^{11}$.

Mais les chances du NEPAD sont moindres dans le sens qu'il est bâtit sur la politique de la main tendue vis-àvis de l'occident.

Depuis plus de 50 ans l'Afrique n'a rien fait pour sortir du sous développement. L'Europe se trouve obligé de préserver ses intérêts sans sentir obligée d'être généreuse envers l'Afrique, parce que les Etats n'ont que des intérêts et pas d'amis.

Donc l'Afrique et son NEPAD peuvent décrier autant de fois la situation politico-économique combien critique qui les caractérise, mais celle-ci semble être éternelle dans la mesure où toutes les initiatives de développement économique prises par les Africains sont battues en brèche.

\section{b. Perspectives}

Plus d'une décennie au moins après sa maternité difficile que faire pour que le NEPAD devienne une stratégie de développement véritable?

Il faudrait :

- $\quad$ Renforcer les politiques de développement au niveau national. Le développement doit être d'abord national avant d'être contributif pour tout le continent. Car le développement ne consiste pas seulement à apprendre comment se servir de la technologie ou d'en fabriquer, mais il concerne tous les aspects de la vie nationale : politique, social et culturel. Rien de ce qui est ancien ne doit forcement être abolis, il s'agira plutôt d'harmoniser les stéréotypes aux nouvelles théories scientifiques, d'où la raison d'être du vieux brocard latin « Historia magistra experentia docet » (l'histoire est la maitresse de l'expérience que nous vivons).

- $\quad$ Que le pouvoir souverain des nations africaines soit indispensable pour maintenir en équilibre et en bon état les formes diverses de développement que préconise le NEPAD. En ceci, le développement de l'Afrique sera, dans une large mesure, une opération nationale des Etats Africains présentement mal gérés.

- Que Les Etats africains renforcent les mécanismes de coopération internationale quoique l'assainissement de leurs structures internes leur incombe en vertu du principe des peuples à disposer d'euxmêmes. Cependant une certaine partie du développement devra être nécessairement fournie par le droit de la coopération internationale. Car le rapport entre l'effort national et l'effort étranger requiert compréhension et doigté de part et d'autre. Ainsi, l'on s'évitera la réalité selon laquelle les pays sous développés sont condamnés à évoluer suivant un rythme uniformément retardé par rapport à la croissance accélérée des pays riches d'où le recours aux importations de capitaux et la course aux investissements étrangers. Ceci est une démonstration incontestable que tout développement ne saurait s'opérer en vase clos et que l'indépendance excluant toute coopération est une utopie parce que le développement a besoin de capitaux privés et publics.

\section{CONCLUSION}

L'Afrique connait tant des problèmes : économique, politique, social et culturel causés par des conflits, l'instabilité macro-économique sur le plan interne et les accords de l'OMC qui le traite injustement. Ceci a pour conséquence l'instabilité du taux de change et du cours des produits de base. Cette inégalité provoque la marginalisation du continent au processus de la mondialisation. Raison pour laquelle le bilan du NEPAD est négatif.

Le seul malheur du NEPAD est celui d'être né de la volonté pour la plupart des dirigeants africains

\footnotetext{
${ }^{11}$ HARSH. E., Art.cit, P.9.
} 
caractérisés par l'absence d'une volonté politique réelle. En outre, le fait pour le NEPAD d'être prédestiné à «La mendicité forcée et planifiée» auprès des institutions occidentales hostiles au développement de l'Afrique, dans un monde où prédominent uniquement les intérêts des Etats puissants et des entreprises multinationales justifie sa maternité difficile. La précipitation des ténors du NEPAD à faire son marketing quelques jours seulement après sa création auprès des partenaires occidentaux, alors que les Africains, bénéficiaires primordiaux des avantages qui découleraient de ce dernier ne le connaissait pas encore ; a facilité son échec processuel. Toutes ces faiblesses trouvent leur force dans les contraintes imposées par l'ordre économique mondial qui fait que l'Afrique se trouve incapable de décider elle-même sur son propre développement. L'exemple de l'ajustement structurel imposé par l'OMC d'une part et d'autre part, les faiblesses internes des structures politiques et socioéconomiques, résultat de la mauvaise gouvernance, ainsi que des guerres et des conflits interethniques à répétition en disent long.

\section{Participation des auteurs}

Les auteurs de la présente étude ont participé tous à la collecte de données et à la rédaction du manuscrit final.

\section{BIBLIOGRAPHIE}

[1] DIOUF A.M, La quête de l'Union et le développement Africain, Paris, L'harmattan, 2003.

[2] HUGON PHILIPPE, L'économie de l'Afrique, Paris, Ed. La découverte, 2000.

[3] KOFFI ANNAN, Rapport sur les causes des conflits et la promotion d'une paix et d'un développement durable en Afrique, 2003.

[4] ESAMBU MATENDA A.B., « Le nouveau partenariat pour le développement de l'Afrique (NEPAD) : un mythe ou une réalité développementalisant dans le contexte de l'Union Africaine » in CAHIER CONGOLAIS D'ETUDES POLITIQUES ET SOCIALES, $\mathrm{n}^{\circ} 25$, Juin 2005, PUL

[5] HARSH E., « Le nouveau partenariat de l'Afrique » in Afrique Relance, $n^{\circ}$ 16, Février 2003

[6] PLATTARD Y., «L'Afrique trente ans après » in Revue de deux mondes, $\mathrm{n}^{\circ}$ 59, Septembre 1991

[7] WENU BECKER, Les organisations internationales africaines et la problématique de la sécurité en Afrique. Praxis de la modélisation d'une approche sécuritaire africaine, thèse de doctorat en R.I., UNILU, 2003-2004.

[8] Nouveau Partenariat pour le Développement de l'Afrique, Octobre 2001.

[9] www.nouvellesdunepad.com, consulté le 28 septembre 2011 